\title{
Notch1 silencing inhibits proliferation and invasion in SGC-7901 gastric cancer cells
}

\author{
GUANGBING WEI $^{1}$, YUANHONG CHANG ${ }^{2}$, JIANBAO ZHENG $^{1}$, SAI HE ${ }^{1}$, \\ NANZHENG CHEN ${ }^{1}$, XIAOLONG WANG ${ }^{1}$ and XUEJUN SUN ${ }^{1}$
}

\author{
${ }^{1}$ Department of General Surgery, First Affiliated Hospital of Medical College, Xi'an Jiaotong University, Xi'an, \\ Shaanxi 710061; ${ }^{2}$ Department of Gastroenterology, Xi'an No. 4 Hospital, Affiliated Guangren Hospital, School of Medicine, \\ Xi'an Jiaotong University, Xi'an, Shaanxi 710004, P.R. China
}

Received September 6, 2013; Accepted January 20, 2014

DOI: $10.3892 / \mathrm{mmr} .2014 .1920$

\begin{abstract}
Downregulation of Notch1 has been shown to exert antineoplastic effects in vivo and in vitro. However, the role of the Notchl gene in the proliferative and invasive ability of gastric cancer cells is not clear. In this study, we investigated the effect of Notchl gene silencing on the proliferation and invasion of gastric cancer SGC-7901 cells. Small interfering RNA (siRNA) targeting Notchl was transfected into SGC-7901 cells using Lipofectamine 2000. Proliferation of SGC-7901 cells was then determined by the MTT assay. Notchl mRNA expression was determined by reverse transcription-polymerase chain reaction (RT-PCR). Invasion of the SGC-7901 cells was detected by the Transwell assay. The protein levels of cyclin D1, cyclin A1 and cyclin-dependent kinase 2 (CDK2) were determined by western blotting. The mRNA levels of matrix metalloproteinase-2 (MMP-2) and cyclooxygenase-2 (COX-2) were determined by RT-PCR. Compared to the control group, the Notchl mRNA level was significantly decreased following transfection. The growth and invasion rates of SGC-7901 cells were significantly reduced after Notchl silencing. Additionally, the expression of cyclin D1 and cyclin A1 proteins and of the $M M P-2$ and $C O X-2$ mRNAs was markedly attenuated. From these results, it was concluded that Notchl gene silencing inhibits the proliferation of gastric SGC-7901 cells by decreasing the expression of cyclins D1 and A1, and reduces the invasive ability of SGC-7901 cells through the downregulation of $M M P-2$ and $C O X-2$ genes. Thus, silencing of the Notch1 pathway may be a novel approach in the treatment of gastrointestinal cancer.
\end{abstract}

Correspondence to: Professor Xuejun Sun, Department of General Surgery, First Affiliated Hospital of Medical College, Xi'an Jiaotong University, 277 West Yanta Road, Xi'an, Shaanxi 710061, P.R. China E-mail:sunxy@mail.xjtu.edu.cn

Key words: gastric cancer, proliferation, invasion, Notch1, small interfering RNA

\section{Introduction}

Gastric cancer is the second most common malignant tumor in the world (1) and the third leading cause of cancer-related mortality in China (2). At present, surgical resection of the primary tumor and control of lymph node metastasis are the main types of treatment for early gastric cancer. There is still no effective treatment for patients with distant metastasis or recurrence. The outcome of unresectable or metastatic gastric cancer is extremely poor, although chemotherapy has been demonstrated to confer a benefit in terms of survival and quality of life (3). Therefore, a better understanding of the molecular mechanism underlying the development and progression of gastric cancer is necessary for developing a more effective treatment for this disease.

The Notch family consists of four Notch proteins (Notch1, 2, 3 and 4), which can be activated by their ligands, the Delta-like (DLL)-1, -3, -4, Jagged-1 and -2 proteins (4). The Notch signaling pathway is evolutionarily conserved and regulates numerous cell processes, including proliferation, differentiation and apoptosis during development and tumorigenesis (5). Notch signaling can be activated by a membrane-bound Notch ligand and alterations of the pathway may cause malignancies, including gastric cancer (6). Once Notch signaling is activated, Notch is cleaved to release intracellular Notch, which is associated with transcriptional factors regulating the expression of target genes (7). Findings of recent studies have demonstrated that the Notch signaling pathway is involved in the development of human malignant tumors, such as breast, lung, pancreas, basal cell and other carcinomas, and seems to function as an oncogene or a tumor suppressor depending on the cellular context $(8,9)$.

Among proteins of the Notch pathway, Notch1 and its ligand DLL1 were found to be expressed in eight gastric cancer cell lines (10); Notch1 is expressed in both premalignant and cancer tissues, especially in tissues of intestinal metaplasia and well-differentiated intestinal gastric cancer tissues. Thus, Notch1 is considered to play an important role in both facilitating the metaplastic transition of gastric epithelial cells and in maintaining the sustained proliferation of intestinalized epithelial cells $(11,12)$. Notch1 expression is significantly higher in gastric cancer compared to healthy gastric tissue and 
correlates with tumor size, differentiation grade, depth of invasion and vessel invasion (13). The three-year survival rate is significantly higher in Notch1-negative than in Notch1-positive patients (13).

The matrix metalloproteinase (MMP) family comprises 23 enzymes which degrade almost all components of the surrounding tissue (14), thus promoting cancer growth and invasion (15). Of all MMPs, MMP-2 is one of the best predictors of the invasive ability of tumor cells. Cyclin D1 is a critical cell cycle regulatory protein, which is required for the progression of cancer cells from the G1 to $\mathrm{S}$ phase $(16,17)$. Cyclooxygenase-2 (COX-2) is a rate-limiting enzyme involved in the conversion of arachidonic acid to prostaglandins and thromboxanes (18). Overexpression of COX-2 is directly associated with various inflammatory diseases and several carcinogenetic processes. COX-2 promotes tumor growth through the induction of angiogenesis, inhibition of apoptosis, by increasing tumor invasiveness, and suppressing the immune response (19). Patients expressing Jagged-1 in gastric cancer tissues had a poor survival rate compared to those with no Jagged-1 expression, and the activation of the Notch1 signaling pathway promoted the progression of gastric cancer, at least in part via the induction of COX-2 expression (20).

Downregulation of Notch1 had antineoplastic effects in vivo and in vitro (21-24), however, the role of the Notchl gene in the proliferative and invasive ability of gastric cancer cells is not clear. In this study, we investigated the role of Notchl in the proliferative and invasive ability of the gastric cancer SGC-7901 cells by examining the protein expression of cyclin D1, cyclin A1, cyclin-dependent kinase 2 (CDK2), and the mRNA expression of $M M P-2$ and $C O X-2$ after silencing of the Notchl gene by small interfering RNA (siRNA). We found that Notch1 silencing inhibits proliferation and invasion in SGC-7901 cells by downregulating the expression of cyclin D1, cyclin A1, $M M P-2$ and $C O X-2$. Our findings may contribute in revealing the molecular mechanism underlying the involvement of Notch1 in gastric cancer and provide a theoretical basis for developing a new treatment for this disease.

\section{Materials and methods}

Materials and reagents. The human gastric cancer cell line SGC-7901 was obtained from the Shanghai Institute for Biological Sciences (Shanghai, China). RPMI-1640, fetal bovine serum (FBS) and trypsin were obtained from HyClone (Logan, UT, USA). 3-(4,5-Dimethylthiazol-2-yl)-2,5diphenyltetrazolium bromide (MTT) and dimethyl sulfoxide (DMSO) were obtained from Sigma-Aldrich (St. Louis, MO, USA). Antibodies were purchased from the following companies: anti-human cyclin D1, cyclin A1 and CDK2 from Santa Cruz Biotechnology, Inc. (Santa Cruz, CA, USA); anti-human $\beta$-actin, goat-anti-mouse $\mathrm{IgG}$, rabbit anti-goat IgG, goat anti-rabbit IgG from Sigma-Aldrich. Notch1 and control siRNAs were obtained from Santa Cruz Biotechnology, Inc. Transwell chambers were purchased from Millipore (Billerica, MA, USA). Lipofectamine 2000 was purchased from Invitrogen Life Technologies (Carlsbad, CA, USA). The PCR mix was obtained from Xi'an Runde Biotechnology Co., Ltd. (Xi'an, Shaanxi, China). PCR primer sets were purchased from DingGuo Biotechnology Co., Ltd. (Beijing, China).
Cell cultures and transfection. The human gastric cancer cell line SGC-7901 was maintained in RPMI-1640 medium supplemented with $10 \% \mathrm{FBS}, 100 \mu \mathrm{g} / \mathrm{ml}$ ampicillin and $100 \mu \mathrm{g} / \mathrm{ml}$ streptomycin. The cells were incubated at $37^{\circ} \mathrm{C}$ in a humidified atmosphere containing $5 \% \mathrm{CO}_{2}$. The cells were divided into three groups for transfection: non-transfected (normal) group, negative control group transfected with a siRNA control (si-control group) and test group, transfected with Notchl siRNA (si-Notch1 group). siRNA sequences were as follows: Notch-1, 5'-GCACGCGGAUUAAUUUGCATT-3' and 5'-UGCAAAUUAAUCCGCGUGCTT-3'; negative control, 5'-UUCUCCGAACGUGUCACGUTT-3' and 5'-ACGUG ACACGUUCGGAGAATT-3'. Transfection was performed following the instructions of the Lipofectamine 2000 kit.

Cell proliferation assays. Cells from the three experimental groups were seeded in 96-well tissue culture plates at a density of 5,000-10,000 cells/well $24 \mathrm{~h}$ prior to serum starvation. After serum starvation for $24 \mathrm{~h}$, the cells were cultured in RPMI-1640 medium supplemented with $10 \% \mathrm{FBS}$ and incubated at $37^{\circ} \mathrm{C}$. After 12, 24, 36, 48, 60 or $72 \mathrm{~h}$, the medium was removed and MTT was added to each well and incubated at $37^{\circ} \mathrm{C}$ for $4 \mathrm{~h}$. Optical densities (OD) were measured at $492 \mathrm{~nm}$ on a microplate reader (BioTec Instruments Inc., Winooski, VT, USA). The proliferation rate was defined as $\mathrm{OD}_{\text {test plate }} / \mathrm{OD}_{\text {control plate }}$. Results from three separate experiments are presented as means $\pm \mathrm{SD}$.

Cell invasion assays. The invasive ability of cells in each group was assessed by a chamber-based invasion assay. The upper surface of a filter (pore size, $8.0 \mu \mathrm{m}$; Millipore) was coated with Matrigel (BD Biosciences, Franklin Lakes, NJ, USA). Prior to treatment, the cells that had reached the log phase of growth were cultured for $24 \mathrm{~h}$ in 6 -well plates in medium containing $1 \% \mathrm{FBS}$. The cells $\left(5 \times 10^{4}\right)$ were suspended in RPMI-1640 medium containing 1\% FBS and then seeded in the top chamber, while the medium containing 20\% FBS was added to the bottom chamber to induce the invasion of the cancer cell line. The Matrigel invasion chamber was incubated for $24 \mathrm{~h}$ in a humidified tissue culture incubator. Non-invading cells were removed from the top of the Matrigel with a cotton-tipped swab. Invading cells on the bottom surface of filter were fixed in methanol and stained with crystal violet (Boster Biological Technology Ltd., Wuhan, China). The invasive ability was determined by counting the number of stained cells under a light microscope. The cell invasion assay was performed in triplicate.

Western blotting assays. SGC-7901 cells were lysed in situ with RIPA buffer (50 mM Tris, $\mathrm{pH} 7.5,150 \mathrm{mM} \mathrm{NaCl,} 0.5 \%$ sodium deoxycholate, $1.0 \%$ Triton X-100, 0.1\% SDS), supplemented with protease inhibitors (Roche Diagnostics, Mannheim, Germany) and phosphatase inhibitors (Sigma-Aldrich). Cell lysates were centrifuged at $12,000 \mathrm{x} \mathrm{g}$ for $20 \mathrm{~min}$ at $4^{\circ} \mathrm{C}$ to remove debris. Proteins $(100 \mu \mathrm{g})$ were separated on a $10 \%$ SDS-PAGE gel and transferred to PVDF membranes (Roche Diagnostics). the PVDF membranes were initially blocked with $5 \%$ nonfat dry milk in Tris-buffered saline (TBS) for $2 \mathrm{~h}$ and incubated with primary antibodies overnight at $4^{\circ} \mathrm{C}$. After washing with TBS and Tween-20 solution (TBST; pH 7.4) five 
$\mathbf{A}$

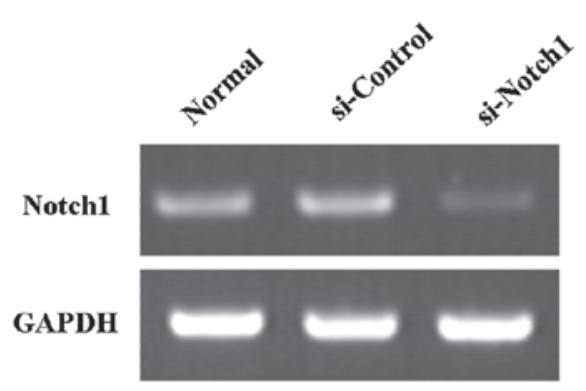

B

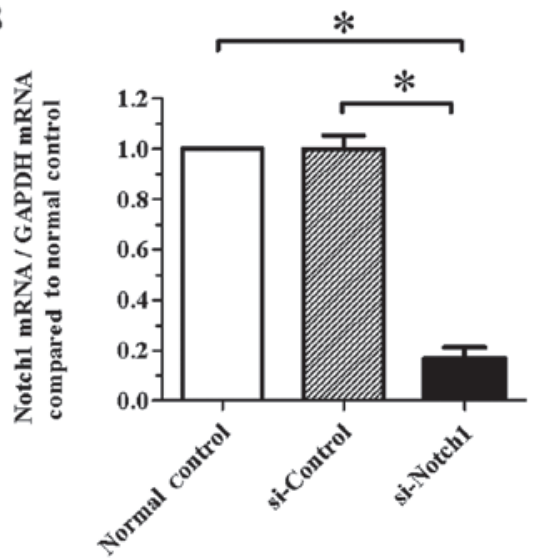

Figure 1. The effect of Notchl-specific targeting with a small interfering RNA (siRNA) was confirmed by reverse transcription-polymerase chain reaction (RT-PCR). (A) RT-PCR showing that after transfection with siRNA for $24 \mathrm{~h}$, the expression of the Notch1 mRNA was reduced. (B) Integrated optical density was measured to evaluate the Notch1 mRNA expression relative to the expression of the glyceraldehyde-3-phosphate dehydrogenase gene (GAPDH), serving as the normalization control. ${ }^{*} \mathrm{P}<0.05$ as compared to the normal group (non-transfected cells).

times, each for $10 \mathrm{~min}$, the PVDF membranes were incubated with HRP-conjugated secondary antibodies at room temperature for $2 \mathrm{~h}$. The membranes were washed again with TBST and an enhanced chemiluminescence (ECL) kit (Gentaur, Santa Clara, CA, USA) was used to develop the immunoblots.

Reverse transcription-polymerase chain reaction (RT-PCR). Total RNA was extracted using TRIzol reagent (Invitrogen Life Technologies) according to the manufacturer's protocol and RT was performed using a PrimeScript ${ }^{\mathrm{TM}}$ RT reagent kit (Takara, Dalian, China). cDNAs (1 $\mathrm{ml}$ for each sample) were amplified by PCR using the primers: Notch1, forward: 5'-GCA GTT GTG CTC CTG AAG AA-3' and reverse: 5'-CGG GCG GCC AGA AAC-3'; MMP-2, forward: 5'-GTG CCC AAA GAA AGG TGC TG-3' and reverse: 5'-AGG AGG GGA GCC ATC CAT AG-3'; COX-2, forward: 5'-ATC CTT GCT GTT CCC ACC CA-3' and reverse: 5'-CTT TGA CAC CCA AGG GAG TC-3'; GAPDH, forward: 5'-GTA AAG ACC TCT ATG CCA TCA-3' and reverse: 5'-GGA CTC ATC GTA CTC CTG CT3-3'. The glyceraldehyde-3-phosphate dehydrogenase $(G A P D H)$ gene served as the normalization control. RT-PCR products were resolved by $1.5 \%$ agarose gel electrophoresis. The results were analyzed and photographed using a UV transilluminator. Each measurement was carried out in triplicate.

Statistical analysis. Results are shown as means \pm standard error. Differences were evaluated with unpaired two-tailed Student's t-tests with unequal variance for multiple comparisons using the SPSS software, version 16.0 (SPSS, Chicago, IL, USA). $\mathrm{P}<0.05$ was considered to indicate a statistically significant difference. Experiments were repeated independently at least three times.

\section{Results}

Expression of Notchl is significantly inhibited by a Notch1-specific siRNA in SGC-7901 cells. In a first set of experiments, we examined the silencing efficiency of a specific siRNA targeting the Notchl gene in SGC-7901 cells. Following cell transfection for $24 \mathrm{~h}$, Notchl silencing was

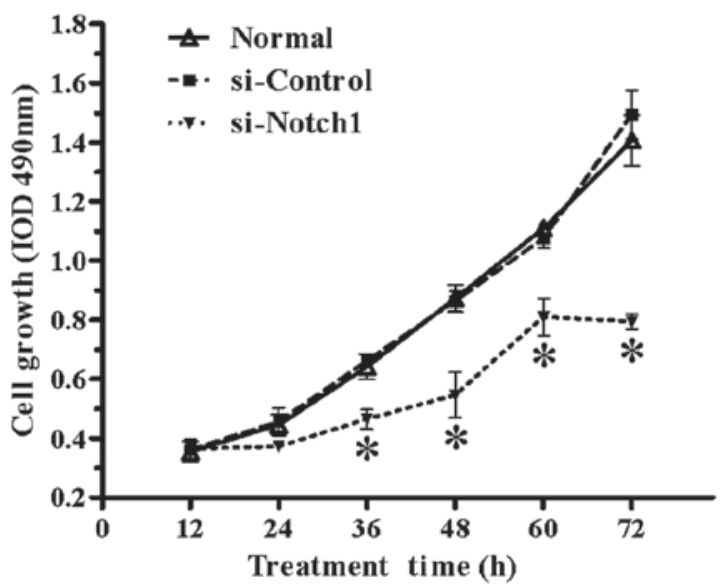

Figure 2. Effect of Notch1 silencing on the proliferation of gastric cancer SGC-7901 cells as assessed by the MTT assay. Mock- (normal) and small interfering RNA (siRNA)-treated SGC-7901 cells were seeded in 96-well tissue culture plates and incubated for the indicated periods. The proliferation rate of the cells in each group was determined by measuring the optical densities (OD) at $492 \mathrm{~nm}$. ${ }^{*} \mathrm{P}<0.05$ as compared to the normal group (non-transfected cells).

confirmed by RT-PCR. As shown in Fig. 1, the mRNA level of Notch1 was significantly reduced in the si-Notch1 group compared to the si-control group $(\mathrm{P}<0.05)$.

Proliferation rate of SGC-7901 cells is significantly impaired by Notch1 silencing. To investigate the effect of Notch1 silencing on gastric cancer cell proliferation, non-transfected and si-RNA-transfected SGC-7901 cells were seeded in 96-well plates, incubated for 12, 24, 36, 48, 60 or $72 \mathrm{~h}$, and their proliferation rate was investigated by the MTT assay. As shown in Fig. 2, the proliferation rate of SGC-7901 cells was markedly reduced by Notchl silencing compared to the normal and si-control groups from $36 \mathrm{~h}$ onwards $(\mathrm{P}<0.05)$.

Effect of Notchl silencing on the expression of cell cycle-related proteins in SGC-7901 cells. The effect of Notch1 silencing on 

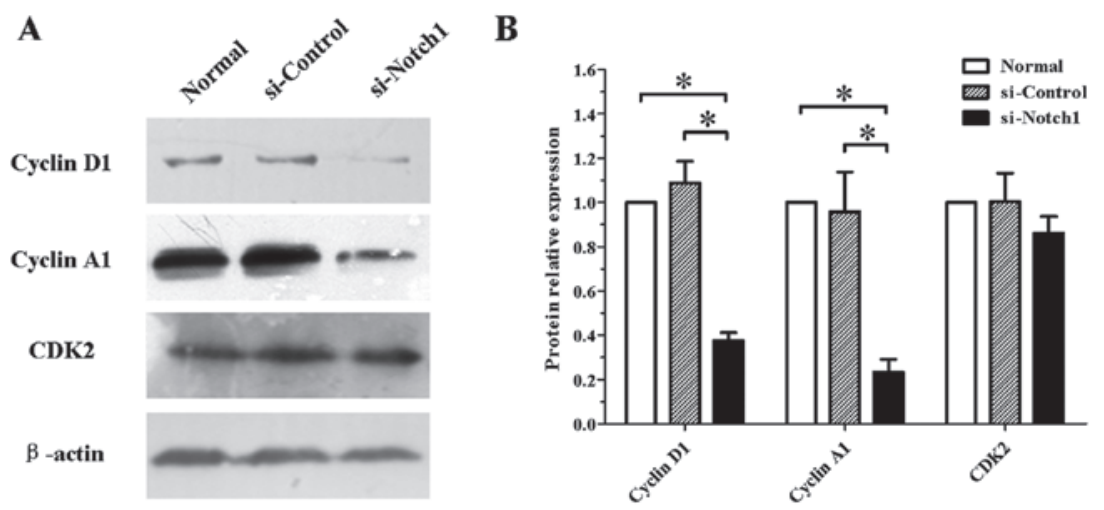

Figure 3. Effect of Notch1 silencing on the expression of cell cycle-related proteins in SGC-7901 cells. (A) Western blotting image after cell tranfection with the Notchl-specific small interfering RNA (siRNA). (B) Optical density was measured to evaluate the protein expression of cell cycle-related proteins, with $\beta$-actin serving as the normalization control. " $\mathrm{P}<0.05$ as compared to the normal group (non-transfected cells).
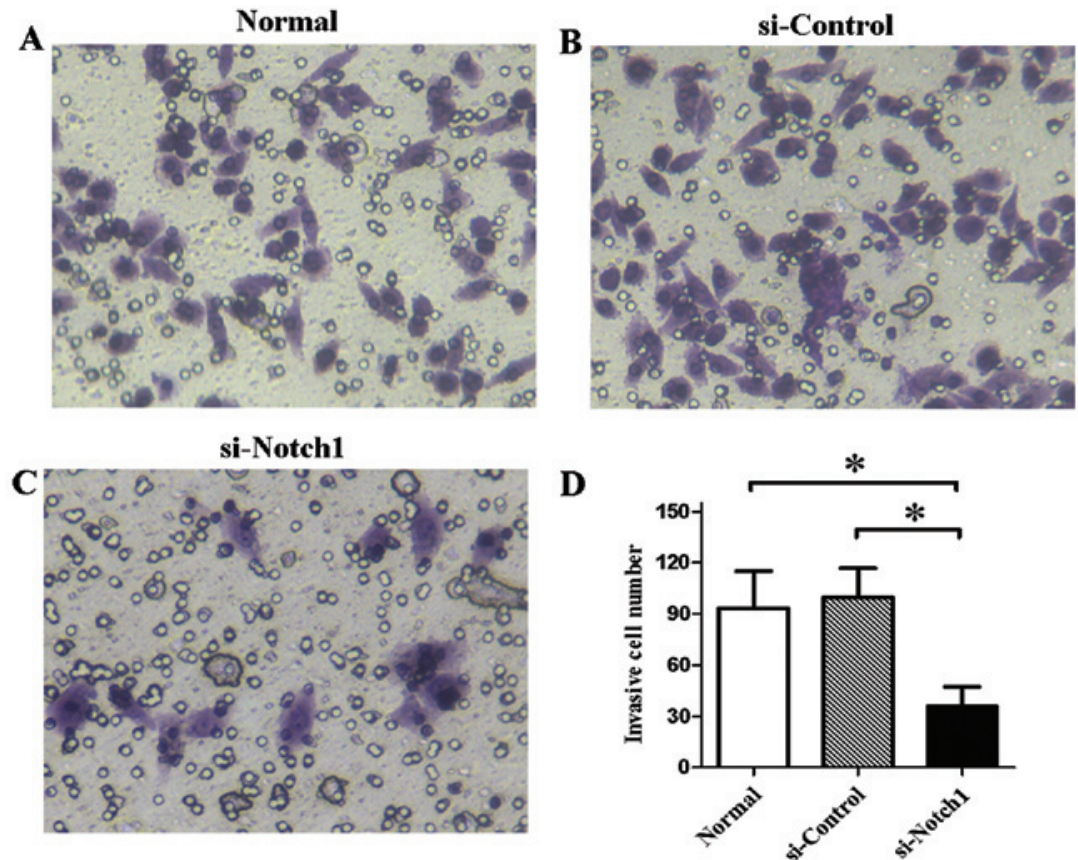

Figure 4. The invasive ability of SGC-7901 cells was inhibited by Notch1 silencing. Microscopic image of cells of the (A) normal control; (B) si-control and (C) si-Notch group and (D) number of invasive cells in each group. " $\mathrm{P}<0.05$ as compared to the normal group (non-transfected cells).
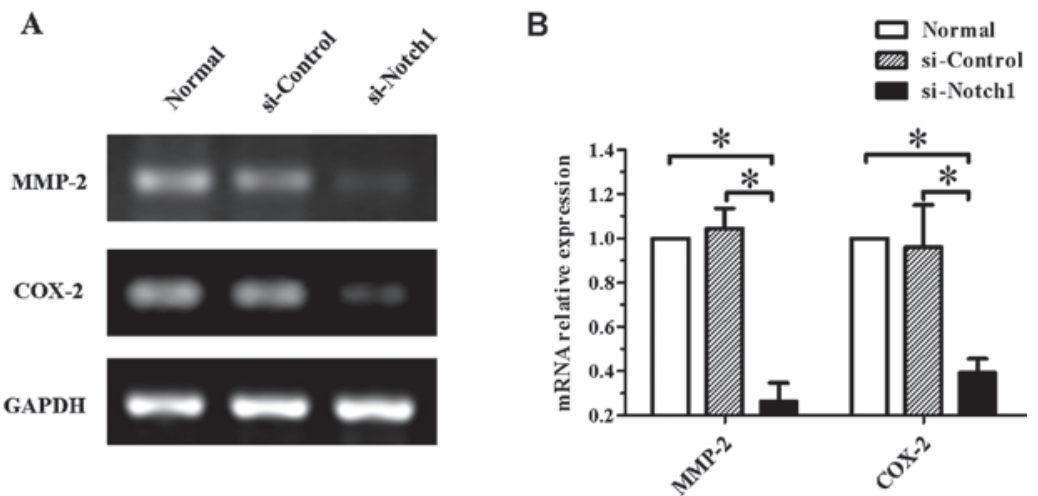

Figure 5. The expression of matrix metalloproteinase-2 (MMP-2) and cyclooxygenase-2 (COX-2) genes is inhibited by Notch1 silencing. (A) Gel image of the reverse transcription-polymerase chain reaction (RT-PCR) result. (B) Optical density was measured to evaluate the mRNA expression of $M M P-2$ and $C O X-2$, with the glyceraldehyde-3-phosphate dehydrogenase gene $(G A P D H)$ serving as the normalization control. " $\mathrm{P}<0.05$ as compared to the normal group (non-transfected cells). 
the expression of cell cycle-related proteins in SGC-7901 cells was assessed. Following transfection with siRNA for $48 \mathrm{~h}$, total cell protein extracts were subjected to western blotting. As shown in Fig. 3, Notchl-silenced SGC-7901 cells showed reduced expression of cyclin D1 and A1. By contrast, the protein expression of CDK2 remained unchanged.

Invasive ability of SGC-7901 cells is inhibited by Notchl silencing. We tested the effect of Notchl silencing on the invasive ability of SGC-7901 cells in vitro. The results of the Transwell assay showed that the number of invasive cells was significantly reduced $(\mathrm{P}<0.05)$ compared to the non-transfected cells or the control siRNA-treated cells (Fig. 4).

Gene expression of MMP-2 and COX-2 is decreased in Notch1-silenced SGC-7901 cells. Since the impairment of the cell invasive ability is commonly due to the modulation of expression of invasion-related genes, to confirm whether the expression of such genes was affected by Notchl silencing, we examined the expression of $M M P-2$ and $C O X-2$ genes. As shown in Fig. 5, the mRNA levels of the two genes were significantly reduced in Notchl-silenced cells $(\mathrm{P}<0.05)$.

\section{Discussion}

To reveal the effect of the Notch1 protein on the proliferative and invasive ability of gastric cancer SGC-7901 cells, we examined the expression of cyclin D1, cyclin A1, CDK2, MMP-2 and COX-2 after silencing of the Notchl gene by siRNA. We found that the Notch1-specific siRNA significantly reduced the expression of the Notchl gene and decreased the expression of cell cycle-related proteins (cyclin D1, cyclin A1 and $\mathrm{CDK} 2)$ and invasion-related genes (MMP-2 and $C O X-2)$, thus attenuating the proliferation and invasion rates of SGC-7901 cells.

The MTT assays demonstrated that silencing of Notchl attenuates the rate of SGC-7901 cell proliferation. We found that the expression of cyclin D1, cyclin A1 and CDK2 was significantly decreased after silencing of the Notchl gene by siRNA, which suggests that Notch signaling mediates the proliferation and differentiation of SGC-7901 cells by directly or indirectly regulating the expression of cell cycle-related genes. A recent study has shown that Notch signaling is involved in the differentiation from gastric epithelium to foveolar glands in normal gastric mucosa (25). It is noteworthy that Notch signaling is associated with glandular differentiation not only in normal gastric mucosa, but also in gastric carcinoma cells. Notch1, 2 and 3 were also detected in human gastric cancer tissue (25). A previous study indicated that Notch1 functions as a tumor-suppressor gene in mammalian skin tissue and that Notchl silencing leads to epidermal and corneal hyperplasia, followed by the development of skin tumors, while it can also promote chemical-induced skin carcinogenesis (26). Furthermore, activation of the Notch1 receptor was shown to facilitate the colony-forming ability and xenografted tumor growth of human pancreatic adenocarcinoma (8).

In this study, inhibition of Notchl gene expression by a specific siRNA led to a significant decrease in the invasive ability of gastric cancer cells, accompanied by the downregulation of $M M P-2$ and $C O X-2$ genes, suggesting that the
Notch/MMP-2/COX-2 signaling pathway regulates the invasive ability of gastric cancer cells by adjusting the expression levels of invasion-related genes. Previous studies suggested that MMPs degrade the extracellular matrix of tumor cells to allow them to invade the surrounding tissue $(27,28)$ and that COX-2 promotes angiogenesis, inhibits apoptosis, increases tumor invasiveness and suppresses immune responses to cause tumorigenesis (19). However, COX-2 expression is an independent prognostic factor of gastric cancer (29).

In conclusion, the silencing of Notchl significantly inhibited the proliferative and invasive ability of the gastric cancer cell line SGC-7901, indicating that the Notch signaling pathway plays an important role in the proliferation and invasion of gastric cancer. Our findings provide a basis for developing new therapies targeting gastric cancer.

\section{Acknowledgements}

This work was financially supported by Grants from the National Natural Science Foundation of China (No. 81172362 and No. 81101874), and the Scientific and Technological Development Research Project Foundation by Shaanxi Province (No. 2012K19-04-02). We are grateful to Dr Xuqi Li (Xi'an Jiaotong University, Xi'an, China) for providing expert opinions on methods of our study and his useful comments. We thank Medjaden Bioscience Ltd. for assisting in the preparation of this manuscript.

\section{References}

1. Siegel R, Naishadham D and Jemal A: Cancer statistics, 2013. CA Cancer J Clin 63: 11-30, 2013.

2. Chen W, Zheng R, Zhang S, Zhao P, Li G, Wu L and He J: Report of incidence and mortality in China cancer registries, 2009. Chin J Cancer Res 25: 10-21, 2013.

3. Oba K, Paoletti X, Bang YJ, Bleiberg H, Burzykowski T, Fuse N, Michiels S, Morita S, Ohashi Y, Pignon JP, et al: Role of chemotherapy for advanced/recurrent gastric cancer: an individual-patient-data meta-analysis. Eur J Cancer 49: 1565-1577, 2013

4. Kopan R and Ilagan MX: The canonical Notch signaling pathway: unfolding the activation mechanism. Cell 137: 216-233, 2009.

5. Borggrefe $\mathrm{T}$ and Liefke R: Fine-tuning of the intracellular canonical Notch signaling pathway. Cell Cycle 11: 264-276, 2012.

6. South AP, Cho RJ and Aster JC: The double-edged sword of Notch signaling in cancer. Semin Cell Dev Biol 23: 458-464, 2012.

7. Dai Q, Andreu-Agullo C, Insolera R, Wong LC, Shi SH and Lai EC: BEND6 is a nuclear antagonist of Notch signaling during self-renewal of neural stem cells. Development 140: 1892-1902, 2013.

8. Mullendore ME, Koorstra JB, Li YM, Offerhaus GJ, Fan X, Henderson CM, Matsui W, Eberhart CG, Maitra A and Feldmann G: Ligand-dependent Notch signaling is involved in tumor initiation and tumor maintenance in pancreatic cancer. Clin Cancer Res 15: 2291-2301, 2009.

9. Bolos V, Mira E, Martinez-Poveda B, Luxan G, Canamero M, Martinez-A C, Manes S and de la Pompa JL: Notch activation stimulates migration of breast cancer cells and promotes tumor growth. Breast Cancer Res 15: R54, 2013.

10. Piazzi G, Fini L, Selgrad M, Garcia M, Daoud Y, Wex T, Malfertheiner P, Gasbarrini A, Romano M, Meyer RL, et al: Epigenetic regulation of Delta-Like1 controls Notch1 activation in gastric cancer. Oncotarget 2: 1291-1301, 2011.

11. Wang Z, Li Y and Sarkar FH: Notch signaling proteins: legitimate targets for cancer therapy. Curr Protein Pept Sci 11: 398-408, 2010.

12. Sun Y, Gao X, Liu J, Kong QY, Wang XW, Chen XY, Wang Q, Cheng YF, Qu XX and Li H: Differential Notch1 and Notch2 expression and frequent activation of Notch signaling in gastric cancers. Arch Pathol Lab Med 135: 451-458, 2011. 
13. Li DW, Wu Q, Peng ZH, Yang ZR and Wang Y: Expression and significance of Notch1 and PTEN in gastric cancer. Ai Zheng 26: 1183-1187, 2007 (In Chinese).

14. Li X, Ma Q, Xu Q, Duan W, Lei J and Wu E: Targeting the cancer-stroma interaction: a potential approach for pancreatic cancer treatment. Curr Pharm Des 18: 2404-2415, 2012.

15. Shuman Moss LA, Jensen-Taubman S and Stetler-Stevenson WG: Matrix metalloproteinases: changing roles in tumor progression and metastasis. Am J Pathol 181: 1895-1899, 2012.

16. McCubrey JA, Steelman LS, Chappell WH, Abrams SL, Wong EW, Chang F, Lehmann B, Terrian DM, Milella M, Tafuri A, et al: Roles of the Raf/MEK/ERK pathway in cell growth, malignant transformation and drug resistance. Biochim Biophys Acta 1773: 1263-1284, 2007.

17. Li T, Kon N, Jiang L, Tan M, Ludwig T, Zhao Y, Baer R and Gu W: Tumor suppression in the absence of p53-mediated cell-cycle arrest, apoptosis, and senescence. Cell 149: 1269-1283, 2012.

18. Cheng HH, Kuo CC, Yan JL, Chen HL, Lin WC, Wang KH, Tsai KK, Guven H, Flaberg E, Szekely L, et al: Control of cyclooxygenase-2 expression and tumorigenesis by endogenous 5-methoxytryptophan. Proc Natl Acad Sci USA 109: 13231-13236, 2012.

19. de Moraes E, Dar NA, de Moura Gallo CV and Hainaut P Cross-talks between cyclooxygenase- 2 and tumor suppressor protein $\mathrm{p53}$ : balancing life and death during inflammatory stress and carcinogenesis. Int J Cancer 121: 929-937, 2007.

20. Yeh TS, Wu CW, Hsu KW, Liao WJ, Yang MC, Li AF, Wang AM, Kuo ML and Chi CW: The activated Notch1 signal pathway is associated with gastric cancer progression through cyclooxygenase-2. Cancer Res 69: 5039-5048, 2009.

21. Portanova P, Notaro A, Pellerito O, Sabella S, Giuliano M and Calvaruso G: Notch inhibition restores TRAIL-mediated apoptosis via AP1-dependent upregulation of DR4 and DR5 TRAIL receptors in MDA-MB-231 breast cancer cells. Int J Oncol 43: 121-130, 2013.
22. Zhou H, Luo Y, Chen JH, Hu J, Luo YZ, Wang W, Zeng Y and Xiao L: Knockdown of TRB3 induces apoptosis in human lung adenocarcinoma cells through regulation of Notch 1 expression. Mol Med Rep 8: 47-52, 2013.

23. Kristoffersen K, Villingshoj M,Poulsen HS and Stockhausen MT: Level of Notch activation determines the effect on growth and stem cell-like features in glioblastoma multiforme neurosphere cultures. Cancer Biol Ther 14: 625-637, 2013.

24. Yabuuchi S, Pai SG, Campbell NR, de Wilde RF, De Oliveira E, Korangath P, Streppel MM, Rasheed ZA, Hidalgo M, Maitra A and Rajeshkumar NV: Notch signaling pathway targeted therapy suppresses tumor progression and metastatic spread in pancreatic cancer. Cancer Lett 335: 41-51, 2013.

25. Kang H, An HJ, Song JY, Kim TH, Heo JH, Ahn DH and Kim G: Notch 3 and Jagged 2 contribute to gastric cancer development and to glandular differentiation associated with MUC2 and MUC5AC expression. Histopathology 61: 576-586, 2012.

26. Nicolas M, Wolfer A, Raj K, Kummer JA, Mill P, van Noort M, Hui CC, Clevers H, Dotto GP and Radtke F: Notch1 functions as a tumor suppressor in mouse skin. Nat Genet 33: 416-421, 2003.

27. Fanjul-Fernandez M, Folgueras AR, Fueyo A, Balbin M, Suarez MF, Fernandez-Garcia MS, Shapiro SD, Freije JM and Lopez-Otin C: Matrix metalloproteinase Mmp-1a is dispensable for normal growth and fertility in mice and promotes lung cancer progression by modulating inflammatory responses. J Biol Chem 288: 14647-14656, 2013.

28. Kaimal R, Aljumaily R, Tressel SL, Pradhan RV, Covic L, Kuliopulos A, Zarwan C, Kim YB, Sharifi S and Agarwal A: Selective blockade of matrix metalloprotease-14 with a monoclonal antibody abrogates invasion, angiogenesis, and tumor growth in ovarian cancer. Cancer Res 73: 2457-2467, 2013.

29. Shi H, Xu JM, Hu NZ and Xie HJ: Prognostic significance of expression of cyclooxygenase-2 and vascular endothelial growth factor in human gastric carcinoma. World J Gastroenterol 9: $1421-1426,2003$ 